\title{
Memantine Upregulates BDNF and Prevents Dopamine Deficits in SIV-Infected Macaques: A Novel Pharmacological Action of Memantine
}

\author{
Falko Meisner', Carsten Scheller', Susanne Kneitz ${ }^{2}$, Sieghart Sopper ${ }^{3}$, Eva Neuen-Jacob ${ }^{4}$, Peter Riederer ${ }^{5}$, \\ Volker ter Meulen' and Eleni Koutsilieri*, ${ }^{*}$, , for the German Competence Network HIVIAIDS \\ Institute of Virology and Immunobiology, University of Wurzburg, Germany; ${ }^{2}$ IZKF (Interdisciplinary Center for Clinical Research), University of \\ Wurzburg, Germany; ${ }^{3}$ German Primate Center, Göttingen, Germany; ${ }^{4}$ Institute of Neuropathology, University of Duesseldorf, Germany; ${ }^{5}$ Clinical \\ Neurochemistry (National Parkinson Foundation Center of Excellence Research Laboratory), Psychiatry and Psychotherapy, University of \\ Wurzburg, Germany
}

$\mathrm{N}$-methyl-D-aspartate (NMDA) receptor activation is involved in the pathogenetic cascades of neurodegenerative disorders including human immunodeficiency virus (HIV) dementia. Memantine, an uncompetitive NMDA receptor antagonist, which has been recently approved for the treatment of Alzheimer's disease, is being discussed as a potential adjunctive therapeutic substance for HIV dementia. We used simian immunodeficiency virus-infected rhesus macaques to assess the effects of memantine on brain dysfunction and brain pathology within 3-5 months after initial infection during early asymptomatic stage of disease. We had shown previously that within this time frame, marked changes were evident in the dopaminergic systems. Memantine was administered two weeks post infection, at peak viremia, in order to prevent early NMDA receptor activation due to immune mediators. We found that memantine prevented onset of dopamine deficits in the brains of SIV-infected macaques, without affecting early brain pathology or peripheral course of infection. Memantine specifically upregulated mRNA and protein expression of the neurotrophic factor brain-derived neurotrophic factor (BDNF), suggesting that the protective effect of memantine on dopamine function may be mechanistically remote from NMDA receptor antagonism. This novel pharmacological action of memantine may also be relevant for other neurodegenerative disorders and supports the involvement of neurotrophic factors in adult brain neuroprotection.

Neuropsychopharmacology (2008) 33, 2228-2236; doi:I0. I038/sj.npp. I 30 I6I5; published online 3I October 2007

Keywords: memantine; dopamine; BDNF; dementia; HIV; SIV

\section{INTRODUCTION}

Infection of the central nervous system (CNS) by human immunodeficiency virus (HIV) type-1 results in neuropsychiatric disorders collectively known as HIV dementia. Although the highly active antiretroviral therapy reduced both the incidence and severity of HIV dementia (McArthur, 2004), the prevalence increased with the patients' life span getting longer and neuropsychological function did not improve in all treated patients (Cysique et al, 2006).

It is currently accepted that macrophages/microglia play a key role in HIV-neurotoxicity through production of viral neurotoxins and/or immune activation with subsequent release of neurotoxic factors acting at the $\mathrm{N}$-methylD-aspartate (NMDA) receptors (Kaul et al, 2001). Most of

*Correspondence: PD Dr Eleni Koutsilieri, Clinical Neurochemistry, Department of Psychiatry and Psychotherapy, University of Wurzburg, Füchsleinstr. 15, Wurzburg 97080, Germany, Tel: + +49 931 20149928, Fax: + + 4993120149553 ,

E-mail: eleni.koutsilieri@mail.uni-wuerzburg.de

Received 26 June 2007; revised 18 September 2007; accepted I October 2007 the evidence that neurons may die due to overactivation of NMDA receptors in HIV dementia comes from in vitro studies on neurotoxic actions of HIV proteins (Mattson et al, 2005) and indirectly from observations of increased levels of products with NMDA-agonistic potential in CSF of HIV-infected patients (Heyes et al, 2001) and simian immunodeficiency virus (SIV)-infected rhesus macaques (Koutsilieri et al, 1999). Additionally, glutamate is increased in brains of asymptomatic SIV-infected animals without being accompanied by neuronal dysfunction (Bossuet $e t a l$, 2004), indicating intact compensatory mechanisms that limit the glutamate-induced neurotoxic cascades during asymptomatic stage of disease. However, the combined mechanisms of increased pools of glutamate with dysregulation of NMDA receptor function due to immune activation can result in neuronal calcium overload and cellular dysfunction and death (Mattson et al, 2005). It is therefore crucial to stop neurochemical alterations before accumulation of toxic products and inflammation are widespread, namely, in early asymptomatic phase of infection.

The NMDA antagonist memantine is an uncompetitive NMDA receptor antagonist with improved voltage dependency, 
kinetics, and affinity compared to endogenous $\mathrm{Mg}^{2+}$ (Danysz and Parsons, 2003) and is currently being used for treatment of senile dementias. Memantine has been tested in a placebo-controlled study for the treatment of sensory neuropathy associated with HIV without beneficial outcome (Schifitto et al, 2006). However, memantine has been shown to exert protective effects on cell toxicity and synaptic functions. Memantine inhibits the HIV gp120evoked calcium changes in neurons and astrocytes and protects neurons from gp120-induced cell death (Lipton, 1992; Nath et al, 2000). Moreover, memantine is beneficial in transgenic mice constitutively expressing gp120 (Toggas et al, 1996) and it improves synaptic function in murine HIV encephalitis (Anderson et al, 2004), indicating that this substance may be a good candidate for an adjunctive therapy for HIV-infected humans.

We found previously extensive impairment in the dopaminergic systems in brains of SIV-infected rhesus macaques during early infection (Jenuwein et al, 2004; Scheller et al, 2005). In this study we hypothesized that early dopamine deficits in brains of SIV-infected macaques may be due to NMDA receptor activation due to inflammation induced by invasion of virus and immune cells in the brain during initial peak brain viremia and we treated monkeys at the peak viremia two weeks after infection with the NMDA antagonist memantine. Our central aim was to assess the protective potential of memantine on the observed early brain dysfunction.

\section{METHODS}

\section{Animals}

Juvenile rhesus monkeys (Macaca mulatta) of Chinese origin were housed individually in indoor facilities on a 12/12 light/dark schedule at the German Primate Center (Göttingen, Germany). Dry food with fresh fruits (bananas and apples) as a dietary supplement was provided twice a day and water was available ad lib. Prior to inoculation, animals were demonstrated to be seronegative for STLV-1, SRV-1 (type D retrovirus), herpes B virus, and SIV. Eleven monkeys were infected under ketamine anesthesia $(10 \mathrm{mg} /$ $\mathrm{kg}$ ) with $100 \mathrm{MID}_{50}(50 \%$ monkey infective dose $)$ of SIVmac251MPBMC. Four SIV-infected animals were treated with memantine $(10 \mathrm{mg} / \mathrm{kg}$, per os) daily. The treatment started at 2 weeks post infection (wpi) and concurrent with peak viremia. All infected animals were killed at predetermined time points without signs of simian AIDS. Four uninfected animals served as controls. Animals were monitored clinically and blood/CSF samplings were performed at regular intervals under ketamine anesthesia from experienced veterinarians in the German Primate Center. Monkeys were killed during the first 5 months of infection in order to investigate early effects of SIV-infection on the CNS. Animal experiments were performed according to the guidelines of Bezirksregierung Braunschweig (604.42502/ 08-02.95) and the revised Directive 86/609/EEC.

\section{Collection and Dissection of Monkey Brain Tissue}

Necropsy was performed at the same time of the day. Anesthetized animals were killed by exsanguination at different time points after infection ranging from 11.9-20.1 wpi. Brains were thoroughly perfused with 21 of RPMI 1640 medium (Gibco, Eggenstein, Germany) containing 3\% fetal calf serum. The brains were quickly removed, partly immersion-fixed (5\% neutral buffered formaldehyde for light microscopy), and partly frozen at $-70^{\circ} \mathrm{C}$ for biochemical analysis. Prior to neurochemical investigations, putamen was dissected on a Teflon plate at $-20^{\circ} \mathrm{C}$ according to a stereotactic atlas using standardized procedures.

\section{BDNF Gene Expression}

Total RNA was extracted from putamen of rhesus macaques using the RNeasy Midi Kit (Qiagen GmbH, Hilden, Germany). The samples were spectrophotometrically scanned from 220 to $320 \mathrm{~nm}$; the A260/A280 of total RNA was typically $>1.9$. RNA quality was tested on the RNAStdsenseChip using the Experion electrophoresis (BioRad, Germany). For all total RNAs extracted, 28S/18S ratios were $>1.5$. Total RNAs were subjected to DNase-I (Qiagen $\mathrm{GmbH}$ ) digestion to remove genomic DNA residues and subsequently cleaned with the RNeasy Midi Kit (Qiagen $\mathrm{GmbH})$. No significant differences between the spectral purity, rate of degradation, molecular size, yield, or tissue $\mathrm{pH}$ between the groups were noted.

\section{Gene Chip Arrays}

For preparation of labeled cRNA, the procedures described in detail in the Affymetrix Gene Chip Expression Analysis Manual (Affymetrix Inc., Santa Clara, CA, USA) were followed. Confirmation of total RNA quality was conducted by hybridizing a small portion of labeled cRNA target to Affymetrix Test3 Array (Affymetrix 900341). Labeled cRNA was hybridized to GeneChip Rhesus Macaque Genome Array (Affymetrix 900656) in an Affymetrix Fluidics Station 400. Hybridized arrays were stained with $10 \mu \mathrm{g} / \mathrm{ml}$ streptavidin-R phycoerythrin (Molecular Probes, Eugene, OR, USA), followed by staining with $3 \mu \mathrm{g} / \mathrm{ml}$ biotinylated goat anti-streptavidin antibody (Sigma Chemical, St. Louis, MO, USA). Arrays were then stained once again with streptavidin- $R$ phycoerythrin and scanned using a GeneChip Scanner 3000. The Gene Chip used contained several probe sets specific for rhesus macaque house-keeping genes such as $\beta$-actin, elongation factor 1 , and GAPDH which served as positive controls. Several murine and yeast probe sets on each chip served as negative controls, and externally spiked bacterial bioB, bioC, bioD, and cre served as positive hybridization controls.

\section{Array Data Interpretation and Analysis}

For data analysis various $\mathrm{R}$ packages were used from the Bioconductor project (www.bioconductor.org). Resulting signal intensities were normalized by variance stabilization (Huber et al, 2002). Quality of all data sets was tested by density plot, RNA degradation plot, and correspondence analysis. Statistical analysis to select differentially expressed genes was performed using the LIMMA (Linear Models for Microarray Analysis) package (Smyth et al, 2003). 


\section{Real-Time RT-PCR}

Isolation of total RNA and real-time PCR was performed as described previously (Jacob et al, 2007). Briefly, total RNA was reverse transcribed using iScriptTM cDNA Synthesis Kit (BioRad Laboratories, Hercules, CA, USA, 170-8890). For qPCR, the iCycler iQ system (BioRad Co., Hercules, CA, USA) with QuantitFast SYBR Green PCR Master Mix (QuantiFast SYBR Green PCR Kit, Qiagen GmbH) was used. PCR was conducted in duplicates for each sample. The following primers were used ie brain-derived neurotrophic factor (BDNF): TTCATAGGAGACACATCCAGC, CACAA AGAGACCACAGCAAGA (Sigma-Genosys, Steinheim, Germany); ribosomal protein L13a (RPL13A), aminolevulinate delta synthase 1 (ALAS1), glyceraldehyde-3-phosphate dehydrogenase (GAPDH), and ribosomal $18 \mathrm{~S}$ were amplified with commercially available specific primer pairs and probes (QuantiTect Custom Assay, Qiagen $\mathrm{GmbH}$ ) and used as internal standards chosen after analysis according to the program geNorm (version 3.3; to be download from http:// medgen31.ugent.be/jvdesomp/genorm/). The amplified transcripts were quantified using the comparative CT method analyzed with the BioRad iCycler iQ system program.

\section{BDNF Protein Concentration}

The putamen was disrupted and homogenized in TEVP, $\mathrm{pH}$ 7.4. (10 mM Tris-HCl, $5 \mathrm{mM} \mathrm{NaF}, 1 \mathrm{mM} \mathrm{Na} \mathrm{NO}_{4}, 1 \mathrm{mM}$ EDTA, $1 \mathrm{mM}$ EGTA) using $5 \mathrm{~mm}$ stainless steel beads (QIAGEN Inc., Valencia, CA, USA) and the TissueLyser system (QIAGEN Inc.). Homogenization was performed at $20 \mathrm{~Hz}$ for $2 \mathrm{~min}$. Homogenates were centrifuged at $32600 \mathrm{~g}$ for $16 \mathrm{~min}$ at $4{ }^{\circ} \mathrm{C}$ to obtain cytosolic fractions. Protein content was determined by the Bradford method using the Biorad Protein Assay (BioRad Co.) and bovine serum albumin as standard.

BDNF concentrations were analyzed by ELISA (Chemicon, Temecula, CA, USA) with a detection range from 7.8 to $500 \mathrm{pg} / \mathrm{ml}$ and no significant crossreactivity with other related neurotrophins. The intra- and interassay coefficients of variation were $\pm 3.7 \%(125 \mathrm{pg} / \mathrm{ml})$ and $\pm 8.5 \%(125 \mathrm{pg} / \mathrm{ml})$, respectively. Samples of the supernatant fraction were diluted to a final protein concentration of $750 \mathrm{ng} / \mu \mathrm{l}$ and a total of $37.5 \mu \mathrm{g}$ of protein was loaded into each well. The plates were read in a Tecan Spectra Mini ELISA reader (Tecan Deutschland GmbH, Crailsheim, Germany) at $405 \mathrm{~nm}$ and data was analyzed by the Synelisa software (Pharmacia \& Upjohn Diagnostics Sverige AB, Uppsala, Sweden).

\section{Measurement of Dopamine, DOPAC, and HVA}

Brain tissues (putamen) were prepared and analyzed with HPLC with electrochemical detection as we previously described (Scheller et al, 2005). Briefly, brain tissues were disrupted in $150 \mathrm{mM}$ phosphoric acid (Merck, Darmstadt, Germany) supplemented with $500 \mu \mathrm{M}$ diethylenetriaminepentaacetic acid by means of a sonifier (Branson W-250, efficacy $50 \%, 40$ pulses) in an argon atmosphere on ice. The homogenates were centrifuged at $44593 \mathrm{~g}$ (Sorvall, Germany), the supernatants were filtered through molecular weight filters (cutoff $5 \mathrm{kDa}$, Millipore, Bedford, USA) at $4^{\circ} \mathrm{C}$, to remove high molecular weight compounds, by spinning down with a MicroRapid centrifuge (Hettich, Tuettlingen, Germany) and stored at $-70^{\circ} \mathrm{C}$ until analysis. Filtrates of $20 \mu$ l were injected into a Rheodyne injector (type 7725, Eppelheim, Germany) and analyzed for dopamine, HVA, and DOPAC by reverse-phase HPLC with electrochemical detection. The HPLC consisted of a solvent delivery pump (P580, Dionex, Germany), an electrochemical detector (EC3000, Recipe, Germany) with a glassy carbon electrode. The detector potential was set at $+750 \mathrm{mV} v s \mathrm{Ag} / \mathrm{AgCl}$, with the sensitivity setting at $5 \mathrm{nA}$. A $5 \mu \mathrm{m}$ analytical column $(250 \times 4.0 \mathrm{~mm}$ nucleosil $120-5 \mathrm{C} 18)$ was used, operating at a flow rate of $1.0 \mathrm{ml} / \mathrm{min}$. The mobile phase consisted of $0.1 \mathrm{M}$ sodium phosphate buffer (Merck) pH 3.35, 84\% (v/ $\mathrm{v})$, and $16 \%(\mathrm{v} / \mathrm{v})$ methanol containing $0.1 \mathrm{mM}$ EDTA (Sigma, St. Louis, USA), $0.65 \mathrm{mM} 1$-octanesulfonic acid, and $0.5 \mathrm{mM}$ triethylamine. Qualitative and quantitative analyses were performed by comparing retention times and peak heights with those of commercially available standards (Sigma).

\section{Histopathology and Immunohistochemistry}

For routine histology, paraffin-embedded sections $(4 \mu \mathrm{m})$ were stained with H\&E, Luxol-fast blue, and Masson's trichrome. In order to disclose activated microglia/macrophages, an antibody against the HLA class II antigen (clone CR3/43, DAKO diagnostica, Hamburg, Germany) was used. Sections were viewed with a BX 50 Olympus microscope, and images were gathered by a CCD camera.

\section{Plasma Viral Load}

Viral RNA in plasma was determined by a quantitative competitive RNA-PCR as we previously described (Scheller et al, 2005). For the target sequence, a highly conserved 267bp region in the SIV gag gene with primer and probe regions homologous for SIVmac, SIVsm, and chimeric SHIV viruses was chosen (Ten Haaft et al, 1998). The detection limit of this assay was 40 RNA equivalents per milliliter of plasma.

\section{Statistical Analysis}

The Mann-Whitney $U$-test was used for statistical analysis. For correlation the Spearman $r$ was determined. The significance level was set at $p<0.05$.

\section{RESULTS}

\section{Animals}

SIV-infected animals did not differ from uninfected controls and SIV/memantine-treated animals with respect to general parameters such as sex or age (Table 1). Infected animals appeared clinically asymptomatic with only a generalized lymphadenopathy. All SIV-inoculated animals became infected and showed a decrease in $\mathrm{CD}^{+}{ }^{+} \mathrm{T}$-cell counts in blood (Table 1). SIV-infected animals showed a disease course typical for slow progressors characterized by a moderate viral load and preservation of memory $\mathrm{CD} 4^{+}$ $\mathrm{T}$ cells (Table 1). They were killed at predetermined time 
Table I SIV Infection in Rhesus Macaques Effects of Memantine on General, Virological, and Immunological Parameters

\begin{tabular}{|c|c|c|c|}
\hline & Uninfected $(n=4)$ & SIV-infected $(n=7)$ & SIV/memantine $(n=4)$ \\
\hline Age (years) & $2.93 \pm 0.53$ & $4.12 \pm 0.18$ & $3.93 \pm 0.4$ \\
\hline wpi & NA & $15.7 \pm 1.4$ & $13.5 \pm 0.58$ \\
\hline Weight (kg) & $3.53 \pm 0.05$ & $4.72 \pm 0.42$ & $3.96 \pm 0.28$ \\
\hline 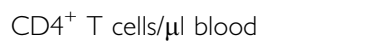 & $984 \pm 145$ & $551 \pm 102 *$ & $510 \pm 137 *$ \\
\hline$\% C D 29 h i / C D 4^{+} T$ cells & $36.4 \pm 1.8$ & $40.8 \pm 3.1$ & $38.9 \pm 5.8$ \\
\hline
\end{tabular}

Abbreviations: NA, not applicable; SIV, semian immunodeficiency virus; wpi, necropsy at weeks post infection.

Data are expressed as means \pm SEM; age, age at time of necropsy; \%CD29hi/CD4 ${ }^{+} \mathrm{T}$ cells, proportion of CD29 high expressing memory cells among CD4 ${ }^{+} \mathrm{T}$ cells; $* P<0.05$ compared to uninfected (Mann-Whitney U-test for nonparametrically distributed values)

points according to the experimental schedule without AIDS-defining conditions. Intrathecal viral antigen levels in these asymptomatic animals were below the detection limit of the assay (data not shown).

\section{Memantine Prevents Dopamine Dysfunction and Upregulates BDNF}

Dopamine levels were reduced in putamen of SIV-infected animals compared to uninfected monkeys during the first 3 months of infection. Metabolite changes were comparable to our previous findings with a larger set of animals (Scheller et al, 2005). However, in the present work these alterations did not reach significance due to the low number of uninfected animals and the relatively high variation in the SIV group (Figure 1). Memantine treatment prevented dopamine deficits in putamen of SIV-infected monkeys (Figure 1a) without statistically significant changes on the metabolites. However, a strong trend was apparent in increased dopamine turnover during SIV infection, which was reversed by memantine (Figure $1 \mathrm{~b}-\mathrm{e}$ ). The great variation in dopamine turnover between animals in the SIV group did not allow a statistically significant difference.

Concomitant with dopamine impairment, BDNF gene expression, and protein concentrations were decreased in SIV-infected animals compared to uninfected controls (Figure 2). Memantine greatly increased BDNF concentrations (Figure 2). The BDNF protein increase was due to an upregulation of BDNF mRNA as determined by gene chip arrays and real-time RT-PCR (Figure $2 \mathrm{a}$ and $\mathrm{b}$ ). A strong correlation was evident between dopamine content and BDNF expression, indicating that expression of BDNF is directly associated with dopamine concentrations in putamen in untreated animals (Figure $2 \mathrm{~d}$ ). Moreover, BDNF levels in infected animals treated with memantine were significantly higher than in uninfected macaques (Figure $2 \mathrm{~b}$ and c).

\section{Memantine Treatment Does Not Alter the Peripheral Course of SIV Infection}

Treatment with memantine did not change the clinical, virological, and immunological findings in SIV-infected animals as viral load in blood, CD4 ${ }^{+}$T-cell numbers, and the proportion of CD29 high expressing memory T-helper cells were similar in both untreated and memantine-treated macaques (Table 1).

\section{Memantine and SIV Brain Pathology}

Occasional perivascular cuffings, as signs of inflammation during early infection, were detected in brain regions in SIV-infected asymptomatic animals. Pathological features of late stage of infection were not evident. Treatment with memantine did not affect either positively or negatively these few early signs of SIV-pathology. The routine histology showed that putamen and in general the basal ganglia remained inconspicuous (Figure 3a and b). Asymptomatic SIV-infected animals exhibited few activated microglia/macrophages, usually in the vicinity of blood vessels (Figure $3 \mathrm{c}$ ). Treatment with memantine did not affect MHCII expression at protein (Figure 3d) or gene levels (data not shown).

\section{DISCUSSION}

The pathogenesis of neurodegenerative disorders including HIV dementia involves excitotoxic processes partly mediated by neurotoxic products released by immunocompetent cells. On the other hand, for HIV dementia it is known that the viral proteins themselves activate NMDA receptors directly and cause neurotoxicity. Among the most vulnerable neurotransmitter systems to HIV neurotoxicity are those regulated primarily by dopamine. Clinical features of HIV dementia are attributed to a dysfunction in dopaminergic systems (Koutsilieri et al, 2002b). Moreover, dopaminergic cell loss is evident in the substantia nigra of AIDS patients (Itoh et al, 2000), and HIV proteins affect dopamine function (Theodore et al, 2006; Wallace et al, 2006). We have shown previously that dopamine deficits constitute initial evidence for brain dysfunction in a susceptible brain region, the putamen in early SIV-infection (Scheller et al, 2005). However, viral load is under detection limit and the putamen of these asymptomatic animals with impaired dopamine neurotransmission is not only spared from histopathological hallmarks of SIV infection but is generally histologically inconspicuous. Immune activation in SIV-infected macaques is evident, however, in the postacute phase of the infection by RNA-profiling (Roberts et al, 2004) and on isolated microglia of SIV-infected macaques 

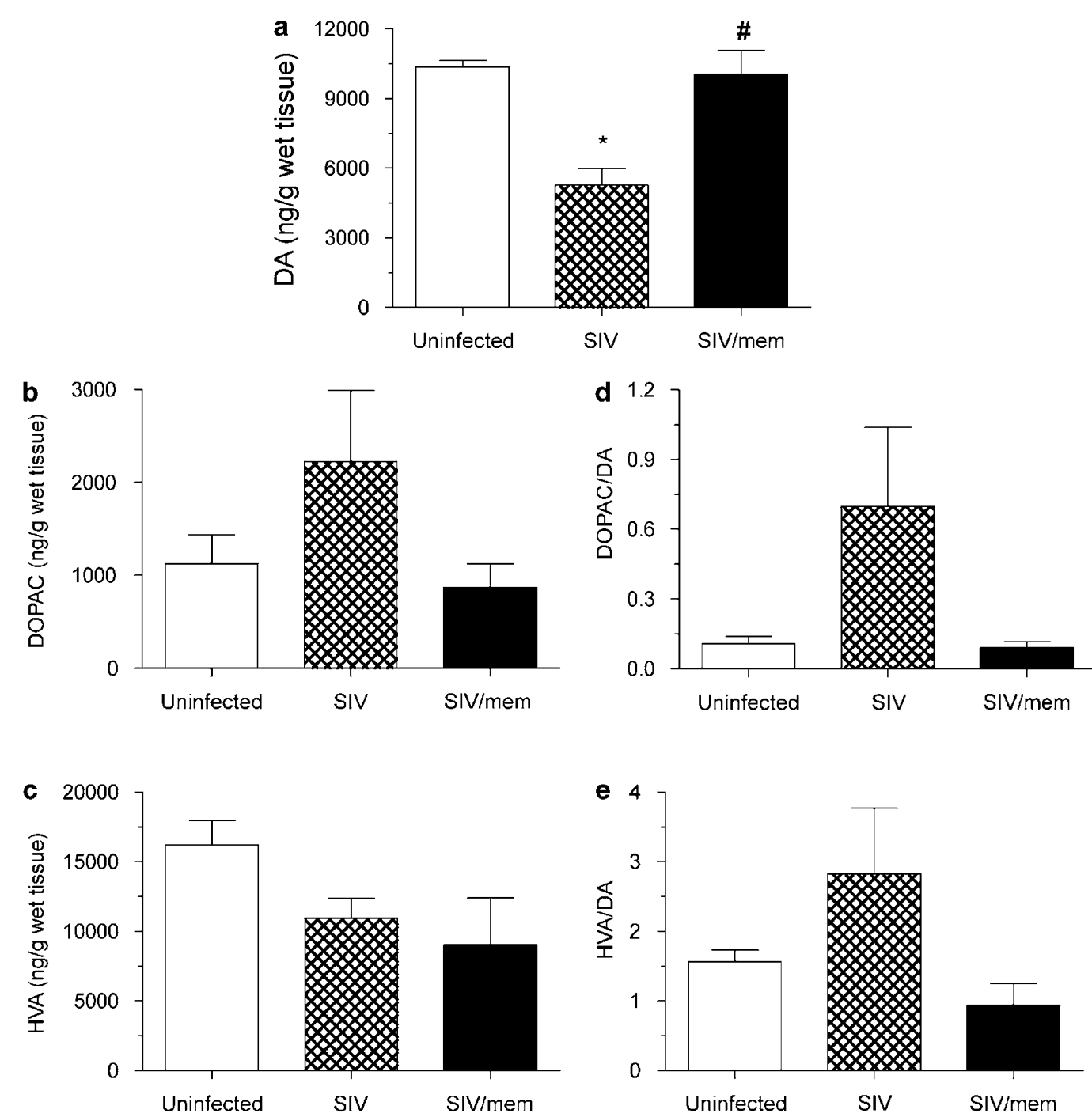

Figure I Memantine restores dopamine deficits in brains of SIV-infected monkeys. Dopamine (a), DOPAC (b), and HVA (c) concentrations in putamen of rhesus monkeys. DOPAC/DA (d) and HVA/DA (e) are ratios between metabolites and dopamine, respectively and represent dopamine turn over. Uninfected, uninfected/untreated $(n=4) ;$ SIV, SIV-infected $(n=7) ; S I V / m e m$, SIV-infected/memantine-treated $(n=4)$. Data represent means \pm SEM, ${ }^{*} p<0.05$, significantly different from uninfected monkeys, \#p<0.05, significantly different from SIV-infected monkeys (Mann-Whitney U-test for nonparametrically distributed values).

(Scheller et al, 2005). Consequently, the neurochemical dysfunction observed may be likely due to indirect toxicity mediated by a transient immune activation after the peak viremia and the subsequent release of detrimental factors or could be due to the direct toxic potential of the viral proteins in that time frame. We hypothesized that early dopamine deficits in brains of SIV-infected macaques may be due to NMDA receptor activation due to initial peak brain viremia and we treated monkeys at the peak viremia with the NMDA antagonist memantine. Memantine completely prevented onset of the dopamine impairment in absence of any obvious effects on viral replication and immune functions. To our knowledge there are no available studies in the literature on the effects of memantine on dopamine and its metabolism neither in healthy nor in diseased brains of rhesus monkeys. Studies in rats provide us with conflicting data. Quack et al (1995) suggested that memantine in rats induces only a modest overflow of dopamine not paralleled by DOPAC and HVA. Hesselink et al (1999) reported no effect of memantine on dopamine in the prefrontal cortex of the rat but an increase in metabolites. On the other hand, two studies investigating the brain region of striatum detected a dose-dependent increase in dopamine and metabolism with microdialysis (Spanagel et al, 1994) and increases in dopamine and DOPAC with pulse voltammetry (Clement et al, 1995). It is obvious from these studies that not only dose of memantine and brain region studied but also methodological issues are responsible for the discrepancy of the studies in the rats. Our study is not directly comparable with the above for several reasons: (a) the rat is known to have a diverse dopamine metabolism from monkey or man. Dopamine is metabolized by both forms of MAO (A and $\mathrm{B}$ ) in the rat brain, MAO-A being the major component (Fowler and Benedetti, 1983), but it is preferentially oxidized by MAO-B in the human brain (Glover et al, 1977) and in rhesus monkeys (Koutsilieri et al, 2002a); (b) the studies concern only extracellular levels of dopamine and metabolites. 
a

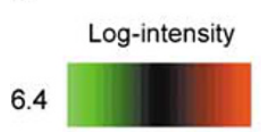

12.2

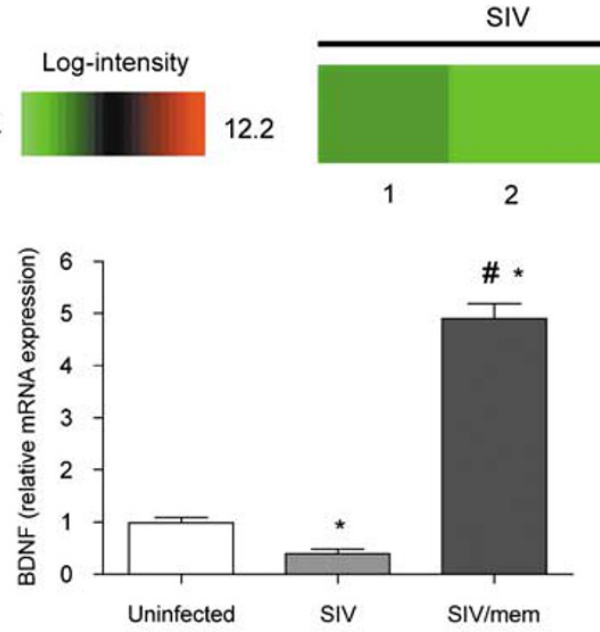

$\mathrm{SIV} / \mathrm{mem}$

b

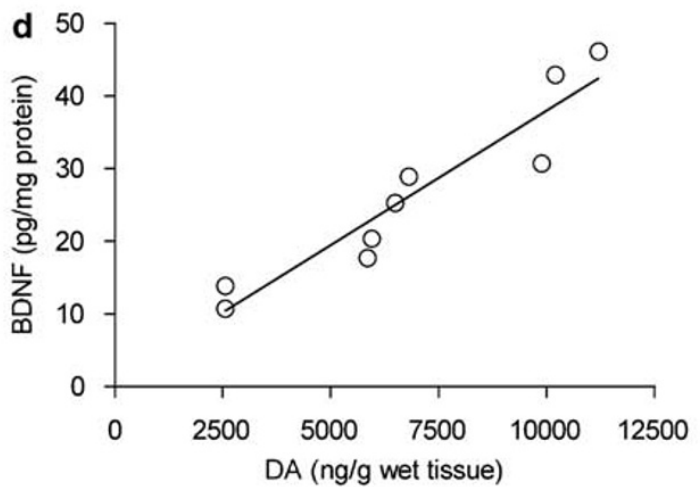

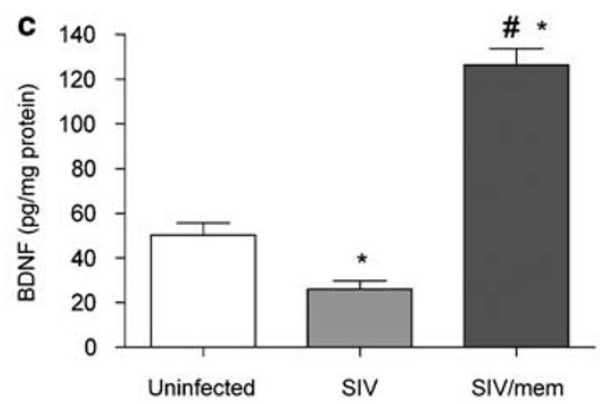

Figure 2 Memantine treatment upregulates brain-derived neurotrophic factor (BDNF) in putamen of SIV-infected monkeys. (a) Heat plot profile of expression levels of BDNF gene exhibits a significant change in basal expression differences between SIV (SIV-infected/untreated, $n=5$ ) and SIV/mem (SIV-infected/memantine-treated, $n=4$ ) groups (gene chip arrays). Samples of each group were pooled and columns I-3 and 4-6 represent triplicates of SIV and SIV/mem groups, respectively. Differences in gene regulation between SIV and SIV/mem is statistically significant ( $p<0.05$, LIMMA Package). (b) Real-time RT-PCR confirms upregulation of BDNF by memantine. (c) BDNF protein concentrations in cytosolic fractions of putamen of rhesus monkeys. Data represent means \pm SEM, $* p<0.05$, significantly different from uninfected, $\# p<0.05$, significantly different from SIV (Mann-Whitney U-test for nonparametrically distributed values). (d) Correlation between BDNF and dopamine concentrations in untreated rhesus monkeys ( $n=9$ from four uninfected and five SIV-infected macaques), Spearman $r=0.99, p<0.05$.

At this point, we should stress out that due to the great differences between the concentration of dopamine in the dopaminergic neuron and in the synaptic cleft, homogenates of brain tissue represent concentrations of dopamine in the intracellular compartment; (c) the brain regions were different.

In contrast to our previous studies showing that the dopaminergic drugs L-DOPA and selegiline exacerbate and accelerate SIV brain pathology (Czub et al, 2004), memantine did not affect adversely the course of SIV brain disease. This supports the idea that adverse effects of L-DOPA and selegiline on the neuropathology and infection were due to excitotoxicity and suggests further the involvement of the NMDA receptor in early SIV infection. On the other hand, the restoration of dopamine deficits by memantine was concomitant with a novel pharmacological action of memantine, the upregulation of the neurotrophic factor BDNF, which has diverse roles. BDNF is responsible for neuron survival, accelerates the development of fast synapses, modulates synaptic efficacy, and it is necessary for the induction and maintenance of long-term potentiation and learning (for review see Guillin et al, 2003). Interestingly, BDNF was recently shown to be significantly reduced in CSF of HIV-infected patients (Albrecht et al, 2006). Moreover, the relation of BDNF to the dopamine synapse has been shown in several studies. BDNF enhances the survival of dopaminergic neurons in mesencephalic cultures (Hyman et al, 1991), regulates the expression of the dopamine receptor D1 (Do et al, 2007) and D3 (Guillin et al, 2003), and on the other side BDNF is reduced in Parkinson's disease, a disease entity with dramatic reduction of dopamine in the putamen (Sawada et al, 2006). Therefore, it is reasonable to think that the amelioration of dopamine deficits by memantine in our study might rather be due to the observed BDNF upregulation than to the generally known NMDA antagonist action of memantine. In this line, a recent report on administration of memantine in adult rats, led to upregulation of several genes independent of the glutamatergic blockade, suggesting broader actions than previously realized (Marvanova et al, 2004). The trophic quality of memantine shown in this manuscript is supported by a paper published during the revision of our manuscript on the effects of a short-term treatment of HIV patients with memantine. In this study memantine enhanced the $\mathrm{N}$-acetyl-aspartate to creatine ratio measured with nuclear magnetic resonance in the brains of the 

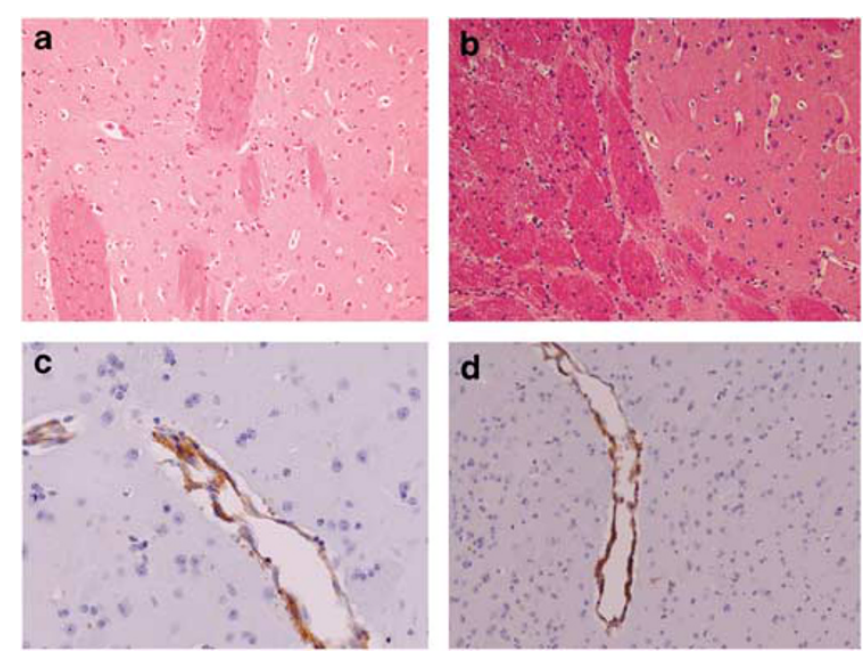

Figure 3 Memantine does not affect early neuropathology in SIV-infected animals. Hematoxylin and eosin (above), HLA class II (clone CR3/43) immunohistochemistry (below). (a) No SIV-specific pathology is evident on routine histology of SIV-infected asymptomatic animal; (b) Treatment with memantine does not change the histological appearance of SIV-infected animal; (c) Few activated CR3/43-positive microglia/macrophages in the vicinity of a blood vessel in SIV-infected asymptomatic animal; (d) Treatment with memantine has no effect on activation of microglia/macrophages in SIV-infected animal.

patients, suggesting the potential to prevent neuronal injury (Schifitto et al, 2007).

The NMDA receptor blocking property of memantine was recognized by the end of the 1980s (Kornhuber et al, 1989). Since then memantine has been shown to prevent neuronal damage in several models of neurodegeneration (Danysz and Parsons, 2003). However, this effect has been always attributed to the inhibition of the NMDA receptor activity. We show here that memantine has also another pharmacological action, which may be dissociated from NMDA antagonism. The induction of BDNF may be due to an effect of memantine on non-neuronal cells known to produce BDNF (Barde, 1994) whereas lack the NMDA receptor signal transduction. Another possibility is that memantine activates extrasynaptic NMDA receptors and promotes neuronal function (Novelli et al, 2005). However, although the correlation between BDNF and dopamine is highly significant, we cannot state here a causative effect and we certainly cannot postulate that the effects of memantine are solely due to BDNF upregulation, but we point to a novel pharmacological action of this agent which has to be considered in the mechanistic sequences of memantine's neuroprotective action in neurodegenerative disorders.

We do not know whether the upregulation of BDNF resulted in an increase in the number of dopaminergic neurons in the midbrain. However, we do know from a previous study of our group that dopamine deficits are not associated with a decrease in density of tyrosine hydroxylase $(\mathrm{TH})$-positive neurons in the midbrains of SIV-infected monkeys during the asymptomatic stage of disease. Only at late stages of infection with development of immunodeficiency, loss of number of TH-positive neurons was evident in our monkeys (Scheller et al, 2005). Although not statistically significant, our results on DA metabolites support the assumption that the increased metabolism of DA in SIV-infected animals can be prevented by memantine and suggest involvement of the primary enzyme for the metabolism of dopamine, MAO in SIV infection. If we now focus on dopamine and BDNF, the most direct explanation of the strong correlation in this study is that BDNFpromoted functional recovery in depressed dopaminergic cells. On the other hand, it is also possible that through actions on the dopamine receptors, BDNF affected the regulation of synthesis of dopamine. Finally, it is reasonable to think that BDNF may have restored dopamine deficits due to structural changes such as sprouting of the dopaminergic neurons. We showed previously that the transcription factor CREB (cyclic AMP response elementbinding protein), which mediates neuronal survival by upregulation of BDNF (Mattson et al, 2004), was reduced in putamen of SIV-infected monkeys and that this correlated with dopamine levels (Scheller et al, 2005). This indicates that an increase in CREB expression may have been involved in upregulation of BDNF levels by treatment with memantine. Additionally, another study showed that BDNF protected cultured cerebellar granule neurons from being killed by the HIV protein Tat through upregulation of the expression of Bcl-2 (Ramirez et al, 2001). Independent of how memantine or BDNF may associate with dopamine function, dopamine preservation is of great significance for HIV dementia pharmacotherapy as there is so far no safe treatment available for the parkinsonian symptoms (Berger and Arendt, 2000) due to the adverse effects through classical anti-parkinson medication (Koutsilieri et al, 2002c).

A very important aspect suggested by our study is the generality of the effects of memantine on the induction of BDNF in other neurodegenerative disease models. Although these disorders have diverse primary causes and brain region targets, the secondary cascades of excitotoxic and inflammatory reactions in which cells of the monocytic lineage play an important role, seem very similar. Several studies in other neurological disease models have to be performed in order to elucidate induction of BDNF by memantine and the mechanistical processes, which are associated with it. On the other hand, there is another issue, which requests attention namely that of the intrinsic effect of memantine on BDNF induction in healthy animals. We did not treat uninfected animals with memantine due to the reduction of monkey numbers in experiments and because our central aim was to assess the neuroprotective role of memantine in the SIV model. However, the intrinsic effect of memantine on BDNF is supported by a study showing that memantine induced BDNF in a short-term $(4 \mathrm{~h})$ treatment in adult rats (Marvanová et al, 2001).

Our results point to a novel pharmacological action of memantine in the diseased and healthy brain. This may be clinically significant not only for HIV dementia but for other neurodegenerative disorders as well and supports the neurotrophic hypothesis in neurodegeneration. As BDNF does not penetrate the blood-brain barrier and therapeutic administration of BDNF can be applied only through invasive techniques, memantine and other similarly acting agents may open new possibilities to regulate the expression of neurotrophic factors in the diseased brain. 


\section{ACKNOWLEDGEMENTS}

We appreciate Christiane Stahl-Hennig's help with the animal experiments. Viral load measurements were performed by Peter ten Haaft. Memantine was provided by Merz Pharmaceuticals GmbH (Frankfurt/Main, Germany).

\section{DISCLOSURE/CONFLICT OF INTEREST}

The study was supported by grants from the 'Gesellschaft für AIDS-Forschung e.V.' and BMBF 01 KI 0211 Competence Network HIV/AIDS. Merz Pharmaceuticals GmbH financially supported the keeping costs of four rhesus monkeys. Peter Riederer received compensation for professional services from Orion Pharma, Espoo, Finland and Schwarz Pharma, Mondheim, Germany (project 'Rotigotine SPM 962') in the previous three years. Sieghart Sopper received compensation for professional services from Glaxo Smith Kline, Research Triangle Park, NC 27709-3398, USA. The rest of the authors declare that, except for income received from the primary employer, no financial support or compensation has been received from any individual or corporate entity over the past three years for research or professional service and there are no personal financial holdings that could be perceived as constituting a potential conflict of interest.

\section{REFERENCES}

Albrecht D, Garcia L, Cartier L, Kettlun AM, Vergara C, Collados L et al (2006). Trophic factors in cerebrospinal fluid and spinal cord of patients with tropical spastic paraparesis, HIV, and Creutzfeldt-Jakob disease. AIDS Res Hum Retroviruses 22: 248-254.

Anderson ER, Gendelman HE, Xiong H (2004). Memantine protects hippocampal neuronal function in murine human immunodeficiency virus type 1 encephalitis. J Neurosci 24: 7194-7198.

Barde YA (1994). Neurotrophins: a family of proteins supporting the survival of neurons. Prog Clin Biol Res 390: 45-56.

Berger JR, Arendt G (2000). HIV dementia: the role of the basal ganglia and dopaminergic systems. J Psychopharmacol 14: 214-221.

Bossuet C, Vaufrey F, Conde F, Chretien F, Pichon J, Hantraye P et al (2004). Up-regulation of glutamate concentration in the putamen and in the prefrontal cortex of asymptomatic SIVmac251-infected macaques without major brain involvement. J Neurochem 88: 928-938.

Clement HW, Grote C, Svensson L, Engel J, Zöfel P, Wesemann W (1995). In vivo studies on the effects of memantine on dopamine metabolism in the striatum and n. accumbens of the rat. J Neural Transm Suppl 46: 107-115.

Cysique LA, Maruff P, Brew BJ (2006). Variable benefit in neuropsychological function in HIV-infected HAART-treated patients. Neurology 66: 1447-1450.

Czub S, Czub M, Koutsilieri E, Sopper S, Villinger F, Muller JG et al (2004). Modulation of simian immunodeficiency virus neuropathology by dopaminergic drugs. Acta Neuropathol (Berl) 107: 216-226.

Danysz W, Parsons CG (2003). The NMDA receptor antagonist memantine as a symptomatological and neuroprotective treatment for Alzheimer's disease: preclinical evidence. Int J Geriatr Psychiatry 18: S23-S32.
Do T, Kerr B, Kuzhikandathil EV (2007). Brain-derived neurotrophic factor regulates the expression of D1 dopamine receptors. J Neurochem 100: 416-428.

Fowler CJ, Benedetti MS (1983). The metabolism of dopamine by both forms of monoamine oxidase in the rat brain and its inhibition by cimoxatone. J Neurochem 40: 1534-1541.

Glover V, Sandler M, Owen F, Riley GJ (1977). Dopamine is a monoamine oxidase B substrate in man. Nature 265: 80-81.

Guillin O, Griffon N, Bezard E, Leriche L, Diaz J, Gross C et al (2003). Brain-derived neurotrophic factor controls dopamine D3 receptor expression: therapeutic implications in Parkinson's disease. Eur J Pharmacol 480: 89-95.

Hesselink MB, De Boer AG, Breimer DD, Danysz W (1999). Dopamine release in the prefrontal cortex in response to memantine following sub-chronic NMDA receptor blockade with memantine: a microdialysis study in rats. J Neural Transm 106: 803-818.

Heyes MP, Ellis RJ, Ryan L, Childers ME, Grant I, Wolfson T et al (2001). Elevated cerebrospinal fluid quinolinic acid levels are associated with region-specific cerebral volume loss in HIV infection. Brain 124: 1033-1042.

Huber W, von Heydebreck A, Sultmann H, Poustka A, Vingron M (2002). Variance stabilization applied to microarray data calibration and to the quantification of differential expression. Bioinformatics 18(Suppl 1): S96-S104.

Hyman C, Hofer M, Barde YA, Juhasz M, Yancopoulos GD, Squinto SP et al (1991). BDNF is a neurotrophic factor for dopaminergic neurons of the substantia nigra. Nature 350: 230-232.

Itoh K, Mehraein P, Weis S (2000). Neuronal damage of the substantia nigra in HIV-1 infected brains. Acta Neuropathol (Berl) 99: 376-384.

Jacob CP, Koutsilieri E, Bartl J, Neuen-Jacob E, Arzberger T, Zander $\mathrm{N}$ et al (2007). Alterations in expression of glutamatergic transporters and receptors in sporadic Alzheimer's disease. J Alzheimers Dis 11: 97-116.

Jenuwein M, Scheller C, Neuen-Jacob E, Sopper S, Tatschner T, ter Meulen V et al (2004). Dopamine deficits and regulation of the cAMP second messenger system in brains of simian immunodeficiency virus-infected rhesus monkeys. J Neurovirol 10: 163-170.

Kaul M, Garden GA, Lipton SA (2001). Pathways to neuronal injury and apoptosis in HIV-associated dementia. Nature 410: 988-994.

Kornhuber J, Mack-Burkhardt F, Riederer P, Hebenstreit GF, Reynolds GP, Andrews HB et al (1989). [3H]MK-801 binding sites in postmortem brain regions of schizophrenic patients. J Neural Transm 77: 231-236.

Koutsilieri E, Scheller C, Czub S, Sopper S, ter Meulen V, Riederer P (2002a). Dopamine is a pathogenetic factor in HIV-induced Neuro-AIDS. In: Nagatsu T, Nabeshima T, McCarty R, Goldstein DS (eds). Catecholamine Research: From Molecular Insights to Clinical Medicine. Kluwer Academic/Plenum Publishers: New York, Boston, Dordrecht, London, Moscow. Adv Behav Biol 53: 475-478.

Koutsilieri E, Sopper S, Heinemann T, Scheller C, Lan J, Stahl-Hennig C, et al (1999). Involvement of microglia in cerebrospinal fluid glutamate increase in SIV-infected rhesus monkeys (Macaca mulatta). AIDS Res Hum Retroviruses 15: 471-477.

Koutsilieri E, Sopper S, Scheller C, ter Meulen V, Riederer P (2002b). Involvement of dopamine in the progression of AIDS Dementia Complex. J Neural Transm 109: 399-410.

Koutsilieri E, Sopper S, Scheller C, ter Meulen V, Riederer P (2002c). Parkinsonism in HIV dementia. J Neural Transm 109: $767-775$.

Lipton SA (1992). Memantine prevents HIV coat protein-induced neuronal injury in vitro. Neurology 42: 1403-1405. 
Marvanová M, Lakso M, Pirhonen J, Nawa H, Wong G, Castrén E (2001). The neuroprotective agent memantine induces brainderivedneurotrophic factor and trkB receptor expression in rat brain. Mol Cell Neurosci 18: 247-258.

Marvanova M, Lakso M, Wong G (2004). Identification of genes regulated by memantine and MK-801 in adult rat brain by cDNA microarray analysis. Neuropsychopharmacology 29: 1070-1079.

Mattson MP, Haughey NJ, Nath A (2005). Cell death in HIV dementia. Cell Death Differ 12(Suppl 1): 893-904.

Mattson MP, Maudsley S, Martin B (2004). BDNF and 5-HT: a dynamic duo in age-related neuronal plasticity and neurodegenerative disorders. Trends Neurosci 27: 589-594.

McArthur JC (2004). HIV dementia: an evolving disease. J Neuroimmunol 157: 3-10.

Nath A, Haughey NJ, Jones M, Anderson C, Bell JE, Geiger JD (2000). Synergistic neurotoxicity by human immunodeficiency virus proteins Tat and gp120: protection by memantine. Ann Neurol 47: 186-194.

Novelli A, Basterrechea MP, Fernandez-Sanchez MT (2005). Glutamate and neurodegeneration. In: Schmidt WJ, Reith ME (eds). Dopamine and Glutamate in Psychiatric disorders. Humana Press: Totowa, NJ, USA, pp 447-472.

Quack G, Hesselink M, Danysz W, Spanagel R (1995). Microdialysis studies with amantadine and memantine on pharmacokinetics and effects on dopamine turnover. J Neural Transm Suppl 46: 97-105.

Ramirez SH, Sanchez JF, Dimitri CA, Gelbard HA, Dewhurst S, Maggirwar SB (2001). Neurotrophins prevent HIV Tat-induced neuronal apoptosis via a nuclear factorkappaB (NF-kappaB)-dependent mechanism. J Neurochem 78: 874-889.

Roberts ES, Burudi EM, Flynn C, Madden LJ, Roinick KL, Watry DD et al (2004). Acute SIV infection of the brain leads to upregulation of IL6 and interferon-regulated genes: expression patterns throughout disease progression and impact on neuroAIDS. J Neuroimmunol 157: 81-92.
Sawada M, Imamura K, Nagatsu T (2006). Role of cytokines in inflammatory process in Parkinson's disease. J Neural Transm Suppl 70: 373-381.

Scheller C, Sopper S, Jenuwein M, Neuen-Jacob E, Tatschner T, Grunblatt E et al (2005). Early impairment in dopaminergic neurotransmission in brains of SIV-infected rhesus monkeys due to microglia activation. J Neurochem 95: 377-387.

Schifitto G, Yiannoutsos CT, Simpson DM, Marra CM, Singer EJ, Kolson DL et al (2006). A placebo-controlled study of memantine for the treatment of human immunodeficiency virus associated sensory neuropathy. J Neurovirol 12: 328-331.

Schifitto G, Navia BA, Yiannoutsos CT, Marra CM, Chang L, Ernst T, et al, The Adult AIDS Clinical Trial Group (ACTG) 301 and 700 Teams and the HIV MRS Consortium (2007). Memantine and HIV-associated cognitive impairment: a neuropsychological and proton magnetic resonance spectroscopy study. AIDS 21: $1877-1886$.

Smyth GK, Yang YH, Speed T (2003). Statistical issues in cDNA microarray data analysis. Methods Mol Biol 224: 111-136.

Spanagel R, Eilbacher B, Wilke R (1994). Memantine-induced dopamine release in the prefrontal cortex and striatum of the rat-a pharmacokinetic microdialysis study. Eur J Pharmacol 262: 21-26.

Ten Haaft P, Verstrepen B, Uberla K, Rosenwirth B, Heeney J (1998). A pathogenic threshold of virus load defined in simian immunodeficiency virus- or simian-human immunodeficiency virus-infected macaques. J Virol 72: 10281-10285.

Theodore S, Cass WA, Maragos WF (2006). Methamphetamine and human immunodeficiency virus protein Tat synergize to destroy dopaminergic terminals in the rat striatum. Neuroscience 137: 925-935.

Toggas SM, Masliah E, Mucke L (1996). Prevention of HIV-1 gp120-induced neuronal damage in the central nervous system of transgenic mice by the NMDA receptor antagonist memantine. Brain Res 706: 303-307.

Wallace DR, Dodson S, Nath A, Booze RM (2006). Estrogen attenuates gp120- and tat1-72-induced oxidative stress and prevents loss of dopamine transporter function. Synapse 59: 51-60. 